\title{
Similar Effects of the Selective Noradrenaline Reuptake Inhibitor Atomoxetine on Three Distinct Forms of Impulsivity in the Rat
}

\author{
Emma SJ Robinson',2, Dawn M Eagle*,', Adam C Mar', Andrea Bari', Gargi Banerjee', Xiaosu Jiang', \\ Jeffrey W Dalley' and Trevor W Robbins' \\ 'Department of Experimental Psychology, Behavioural and Clinical Neurosciences Institute, University of Cambridge, Cambridge, UK
}

\begin{abstract}
Atomoxetine is a noradrenaline-specific reuptake inhibitor used clinically for the treatment of childhood and adult attention deficit hyperactivity disorder (ADHD). Studies in human volunteers and patient groups have shown that atomoxetine improves stop-signal reaction time (SSRT) performance, an effect consistent with a reduction in motor impulsivity. However, ADHD is a heterogeneous disorder and it is of interest to determine whether atomoxetine is similarly effective against other forms of impulsivity, as well as the attentional impairment present in certain subtypes of ADHD. The present study examined the effects of atomoxetine on impulsivity using an analogous SSRT task in rats and two additional tests of impulsivity; delay discounting of reward and the five-choice serial reaction time task (5CSRTT), the latter providing an added assessment of sustained visual attention. Atomoxetine produced a significant dosedependent speeding of SSRT. In addition, atomoxetine produced a selective, dose-dependent decrease in premature responding on the 5CSRTT. Finally, on the delay-discounting task, atomoxetine significantly decreased impulsivity by increasing preference for the large-value reward across increasing delay. These findings conclusively demonstrate that atomoxetine decreases several distinct forms of impulsivity in rats. The apparent contrast of these effects with stimulant drugs such as amphetamine and methylphenidate, which generally act to increase impulsivity on the 5CSRTT, may provide new insights into the mechanisms of action of stimulant and nonstimulant drugs in ADHD.
\end{abstract}

Neuropsychopharmacology (2008) 33, I028-1037; doi:I0.1038/sj.npp. I 30 I487; published online I8 July 2007

Keywords: impulsivity; attention; stop signal reaction time task; five-choice serial reaction time task; delay discounting

\section{INTRODUCTION}

Atomoxetine (tomoxetine, LY 139603) is a selective noradrenaline (NA) reuptake blocker (Bymaster et al, 2002; Swanson et al, 2006) used clinically to treat both adult and childhood attention deficit hyperactivity disorder (ADHD) (Kratochvil et al, 2003, 2006; Wilens, 2006). Unlike other medications used in ADHD, such as amphetamine and methylphenidate, atomoxetine has no appreciable affinity for dopamine (DA) receptors or the DA transporter, although it may indirectly boost prefrontal cortical DA via its effects on the NA transporter (Bymaster et al, 2002). Atomoxetine also displays low affinity for the serotonin

\footnotetext{
*Correspondence: Dr DM Eagle, Department of Experimental Psychology, Behavioural and Clinical Neuroscience Institute, University of Cambridge, Downing Street, Cambridge CB2 3EB, UK, Tel: + 44 I 223765 292, Fax: + 441223333564 ,

E-mail: del02@cam.ac.uk

${ }^{2}$ Current address: Department of Pharmacology, School of Medical Sciences, University Walk, Bristol BS8 ITD, UK.

Received 26 January 2007; revised 23 April 2007; accepted 17 May 2007
}

(5-HT) transporter with a dissociation constant of $77 \mathrm{nM}$ compared with $5 \mathrm{nM}$ for NA transporter and $1451 \mathrm{nM}$ for the DA transporter (Bymaster et al, 2002).

Recently, atomoxetine has been shown to improve performance in normal human volunteers in a stop-signal reaction time (SSRT) task (Chamberlain et al, 2006), which provides a measure of motor inhibition and the propensity toward behavioral impulsivity (Logan and Cowan, 1984). Deficits in SSRT task performance are commonly found in disorders of behavioral inhibition, such as ADHD, Parkinson's disease (Gauggel et al, 2004), schizophrenia (Badcock et al, 2002; Rubia, 2002; Rubia et al, 2001), obsessivecompulsive disorder (Krikorian et al, 2004), and chronic cocaine (Fillmore and Rush, 2002) or methamphetamine use (Monterosso et al, 2005). SSRT itself is especially susceptible to damage to the right inferior frontal cortex in humans (Aron et al, 2003a; Rubia et al, 2003), and in rats following damage to the ventral orbitofrontal cortex, but not the prelimbic or infralimbic prefrontal cortex (PFC) (Eagle and Robbins, 2003b; Eagle et al, 2007a).

SSRT represents the time taken to inhibit an already initiated motor response. SSRT performance in ADHD is 
improved, in both humans and rodents, by stimulants such as methylphenidate (Tannock et al, 1989; Aron et al, 2003b; Eagle et al, 2007b) and by the anti-narcoleptic agent modafinil (Turner et al, 2003, 2004; Eagle et al, 2007b). A recent study in rats also demonstrated the ability of atomoxetine to reduce impulsive responding on a fivechoice serial reaction time task (5CSRTT) (Blondeau and Dellu-Hagedorn, 2006).

Numerous studies in rats indicate that different forms of impulsivity can be dissociated to some extent, however, in relation to the effects of stimulants, very few studies have used low doses in the ranges that are associated with therapeutic effects in ADHD (Berridge et al, 2006). For example, D-amphetamine impairs the ability to wait, as shown by its disruptive effects on DRL schedule performance (Bizot, 1998) and in increasing premature responding on the 5CSRTT (Cole and Robbins, 1987, 1989). However, depending on dose and precise testing conditions, amphetamine can either reduce or enhance impulsive choice in delayed discounting situations (Cardinal et al, 2000; Richards et al, 1999; Winstanley et al, 2006). Moreover, D-amphetamine and methylphenidate generally improve SSRT performance, although their effects can be baseline dependent (Boonstra et al, 2005; Feola et al, 2000; Eagle and Robbins, 2003a; Eagle et al, 2007b). There is also evidence that the various tests of impulsivity can be dissociated in terms of underlying neural substrates. For example, lesions of the nucleus accumbens core region impair delay discounting by enhancing impulsive choice (Cardinal et al, 2001) but have no effect on SSRT (Eagle and Robbins, 2003b). By contrast, lesions of the medial striatum impair SSRT (Eagle and Robbins, 2003a) and enhance premature responding on the 5CSRTT (Rogers et al, 2001), while reducing impulsive choice (DM Eagle, unpublished observations). The effects of central 5-HT depletion also differ across the various measures of impulsivity (Winstanley et al, 2005; DM Eagle, unpublished observations).

In the present study we compared the effects of systemic atomoxetine in three different paradigms for measuring impulsivity; the SSRT task, the 5CSRTT, and delay discounting of reward. For the SSRT task, in addition to examining the overall, dose-dependent effects of atomoxetine, we also subdivided the group into slow- and faststopping subgroups to determine whether the effects of atomoxetine on SSRT were baseline dependent, as reported previously for other ADHD medications (Feola et al, 2000; Eagle et al, 2007b). The 5CSRTT also allows for the measurement of attentional capacity because of its requirement for rats to detect brief visual targets (Robbins, 2002). As attentional function, as well as impulsivity, is known to be affected in certain subtypes of ADHD, the use of these three behavioral procedures provides a comprehensive and directly comparable approach to assessing the behavioral effects of atomoxetine, with methods analogous to those used in human studies (cf. Chamberlain et al, 2006).

\section{MATERIALS AND METHODS}

\section{Subjects}

The subjects were male Lister-hooded rats weighing approximately $250 \mathrm{~g}$ at the start of training and $350-450 \mathrm{~g}$ at the start of dosing (Charles River, UK), housed in pairs or groups under temperature-controlled conditions and $12: 12 \mathrm{~h}$ light-dark cycle (lights off at 0700). They were maintained at approximately $90 \%$ of their free feeding weight by restricting access to laboratory chow (Purina, UK) to $18 \mathrm{~g}$ per day per rat. Water was provided ad libitum. All procedures were conducted in accordance with the requirements of the UK Animals (Scientific Procedures) Act 1986 and in accordance with local institutional guidelines. All behavioral testing was carried out between 0800 and 1700 during the animals' active phase.

\section{Drugs}

Atomoxetine hydrochloride was kindly provided by Eli Lilly and dissolved in $0.01 \mathrm{M}$ phosphate-buffered saline. Drugs were prepared fresh each day and administered by i.p. injection at a dose volume of $1 \mathrm{ml} / \mathrm{kg}$.

\section{SSRT Task for Rats}

All sessions were performed in six operant chambers (Med Associates, VT, USA) as described previously (Eagle and Robbins, 2003a). Control of the chambers and online data collection were conducted using the Whisker control system (Cardinal and Aitken, 2001), with the Cambridge Stop Task program, written by DM Eagle and JMC England (2002).

\section{Stop-Signal Reaction Time Task}

Rats were trained to perform the SSRT task following a training program that has been previously described in detail (Eagle and Robbins, 2003a; Eagle et al, 2007b). In all sessions, trials were initiated with a nose poke to the central food well, after which, the left lever and left light were presented. A press on the left lever resulted in the right lever and right light being presented, and the left lever and left light were withdrawn/extinguished. If a rat failed to press the left lever within $30 \mathrm{~s}$, the left lever was withdrawn, rats received a 5-s timeout, and the trial was recorded as an omission trial. Rats were trained to perform a rapid reaction time (RT) response from left lever to right lever-the go response. Response speed was maintained by limiting the time for which the right lever was presented - the limited hold (LH), maintained at a constant value for each rat throughout the study. Study groups were matched for LH. During go trials, rats were rewarded with a pellet delivered to the central food well for pressing the right lever but received a timeout of $5 \mathrm{~s}$ in darkness if they failed to press the right lever within the $\mathrm{LH}$ period.

On $20 \%$ of the trials, the stop trials, a tone $(40 \mathrm{~ms}$, $4500 \mathrm{~Hz}$ ) was presented at a predetermined time between the left- and right-lever presses. Stop trials were presented randomly within the session to discourage the rats from anticipating presentation of the stop trials. On stop trials, the rats were required to initiate the same response as on go trials, but after hearing the stop signal, the rat was required to stop completion of the go response, ie to refrain from pressing the right lever. The rat was required to withhold from responding for the $\mathrm{LH}$ period, after which it was rewarded with a pellet. An incorrect response, which was a press on the right lever, resulted in a timeout of $5 \mathrm{~s}$ of 
darkness. On a few trials designated as stop trials, the rat responded on the right lever before the onset of the tone (more common for late tone presentations), and these trials were reclassified as go trials to maintain the overall proportion of valid stop trials in each session at $20 \%$.

\section{Exclusion Criteria and Selection Procedure}

To apply Logan's race model (Logan, 1994) to behavioral data, rats must perform go trials as quickly as possible while attempting to stop on all stop trials after the stop signal is detected. Failure to perform the task in this way may be reflected in the form of the inhibition function and go reaction time (GoRT) across different SSDs. Therefore, rats were excluded from further analysis if they showed inverted inhibition functions (accuracy of stopping improved as the stop signal was played closer to the go signal), if go accuracy was inversely correlated with stop accuracy, or if GoRT systematically increased with SSD (more usually presented as a change in go-trial accuracy in the rat SSRT task). Such behavioral patterns reflect strategic changes in performance that cannot be accommodated by the race model. All rats were tested across a full range of SSDs, their inhibition functions were plotted, and SSRT calculated.

To investigate inhibition functions after initial training, rats first received $20 \mathrm{~min}$, 200-trial baseline sessions, during which, the stop signal was presented as the left lever was pressed (ie with no delay between the onset of the go response and the presentation of the stop signal). Mean GoRT and SSDs for each rat were calculated from three no-delay baseline sessions. To investigate individual rat inhibition functions, over the following five sessions (20 min, 200 trials), inhibition functions were generated with SSDs presented in a randomized order from the following set: $\mathrm{SSD}=\mathrm{GoRT}-600 \mathrm{~ms}$; GoRT $-500 \mathrm{~ms}$; GoRT $-400 \mathrm{~ms}$; GoRT-300 ms; GoRT-200 ms.

On days where SSRT was calculated (SSRT sessions for drug-testing days and baselines), rats performed one session per day that was divided into $3 \times 10$-min test periods (StopA, StopB, and StopC), each period with a maximum of 80 trials. In StopA, on stop trials there was no delay between the start of the go trial (left lever press) and the onset of the stop signal. Data from StopA were used to calculate GoRT for each rat, and this GoRT was used to set the stop-signal delays in periods StopB and StopC. StopB presented stop signals at GoRT-500 ms and Stop C presented stop signals at GoRT-300 ms. SSRT was estimated from the data from StopB and StopC. These methods are adapted from the original methods of Eagle and Robbins (2003a).

\section{Testing Procedure}

Testing was carried out using an incremental dose response design with a postdrug baseline session included in the analysis to check that effects observed were due to drug treatment and not a result of a change in baseline across the period of testing. Animals received weekly dosing with atomoxetine $(0,0.6,1.0$, and $1.8 \mathrm{mg} / \mathrm{kg}$ in $0.01 \mathrm{M}$ phosphatebuffered saline) administered by i.p. injection, $30 \mathrm{~min}$ before testing.

\section{Five-Choice Serial Reaction Time Task}

A detailed description of the nine-hole apparatus (Carli et al, 1983) and procedures (Dalley et al, 2004) has been provided previously. The boxes were controlled by Whisker software (Cardinal and Aitken, 2001). Subjects were trained to detect the location of a brief visual stimulus $(0.5 \mathrm{~s}$ in duration) presented pseudorandomly in one of five apertures over a large number of trials, as described previously (Dalley et al, 2004). A number of performance measures were recorded, including choice accuracy (the proportion of correct responses to the total number of correct and incorrect responses), omissions (a failure to respond within the intertrial interval (ITI) and a short period thereafter), premature responding (responses made before the target stimulus), correct response latency (the time from the stimulus onset to a correct response), and magazine latency (the time from a correct response to the collection of food in the magazine). Subjects were considered to have acquired the task when their accuracy was greater than $80 \%$ and omissions were fewer than $20 \%$.

\section{Testing Procedure}

Once animals reached criterion, baseline performance was stabilized for at least 15 further sessions. All experimental testing was carried out using a within-subject study design with drug treatments administered using a fully randomized Latin square. Animals received baseline sessions on Mondays and Thursdays, drug dosing was carried out on Tuesday and Friday, and animals had Wednesday and the weekend off. Animals were tested under baseline conditions (stimulus duration $0.5 \mathrm{~s}$, ITI $5 \mathrm{~s}$, and LH $5 \mathrm{~s}$ ) with atomoxetine $(0,0.6,1.0$, and $3 \mathrm{mg} / \mathrm{kg}$ in $0.01 \mathrm{M}$ phosphatebuffered saline) administered by i.p. injection, $30 \mathrm{~min}$ before testing.

\section{Delay-Discounting Paradigm}

Testing was performed in six operant chambers (Med Associates) with identical dimensions and configuration to those used in the SSRT task. For the first two training phases of the experiment, control of the chambers and online data collection were conducted using an operant control software client (developed by AC Mar) written in Visual Basic 6.0 to run on the Whisker control server (Cardinal and Aitken, 2001). The main delay-discounting task phase was performed using the Impulsive Choice software client, which is included in the Whisker software suite (Cardinal and Aitken, 2001).

Training was conducted over three phases with only slight modifications from experimental procedures previously described (Cardinal et al, 2000; Winstanley et al, 2005; see Mar and Robbins, 2007 for greater detail). In the first training phase, rats were trained to press the left and right levers to receive a food pellet (Noyes dustless pellets, $45 \mathrm{mg}$; Sandown Scientific, UK). Each session in this phase consisted of a maximum of 100 trials or $30 \mathrm{~min}$. Subjects were trained for 3-4 sessions until all had reached a criterion of at least 60 reinforced trials with at least 30 on each lever. In the second phase rats were trained to initiate trials after a requisite ITI with all levers retracted and both 
the house and stimulus light turned off. Each session consisted of 90 trials spaced every $40 \mathrm{~s}$ (60 min total). Failure to nose poke within $10 \mathrm{~s}$ aborted the trial and initiated the ITI. Subjects were trained for three sessions until all had reached a criterion of at least 60 reinforced trials with at least 30 on each lever.

The final phase comprised the main delay-discounting procedure. Each session consisted of 60 trials, divided into five blocks of 12 trials, with trials spaced every $100 \mathrm{~s}$. Each 12-trial block began with 2 'forced' trials in which only one lever was presented (left or right in a random presentation order for every pair of trials), followed by 10 'choice' trials in which both levers were presented. Each rat had one lever designated as the 'immediate' lever (always associated with an immediate, single food pellet) and one lever as the 'delay' lever (always associated with four pellets, but delivered after a delay that was increased between each of the five 12-trial blocks (0,10,20,40, and $60 \mathrm{~s})$. Levers assignments were counterbalanced across rats. Choice ratios-delayed lever presses/total lever presses - were calculated for each rat at each delay using the choice trial responses (ie excluding single lever trials) summed across three consecutive sessions. Using the data from two contiguous three-session blocks, a two-way repeated measures analysis of variance (ANOVA) was performed with choice ratios as the dependent variable and three-Session Block and Delay as within-subjects factors. Two criteria were used to assess stability of performance by applying this ANOVA to successive, contiguous three-session blocks (Mar and Robbins, 2007). The first criterion was judged to be met when no significant main effect or interaction with threeSession Block $(\alpha<0.05)$ was found; an indication of the consistency of the group's behavior. The second criterion was judged to be met when there was a significant main effect of Delay $(\alpha<0.01)$, where pairwise comparisons show smaller choice ratios for at least some of the longer delays; an indication of sensitivity to delays (Cardinal et al, 2000). Performance was considered stable when both criteria were met in the same contiguous three-session blocks. Subjects were trained for 33 sessions, which were 9 sessions past the point at which most rats had reached the two criteria.

\section{Testing Procedure}

The behavioral testing procedure was identical to that employed in the third phase of training. Four doses of atomoxetine $(0,0.3,1$, and $3 \mathrm{mg} / \mathrm{kg}$ in $0.01 \mathrm{M}$ phosphatebuffered saline) were counterbalanced using a fully randomized Latin-square design. Each of the four dosing sessions was separated by a washout day (no drugs or testing) and a drug-free baseline test session. All doses were administered by i.p. injection $30 \mathrm{~min}$ before behavioral testing.

\section{Statistical Analysis}

Data from each of the three tasks were analyzed by ANOVAs using SPSS 12.0.1 (Chicago, IL, USA). Graphs were plotted using Graphpad Prism 4.0 (Graphpad Software, USA). All tests of significance were performed at $\alpha=0.05$, and models were full factorial unless otherwise stated. Homogeneity of variance was verified using Levene's test.
For repeated measured analyses, Mauchly's test of sphericity was applied and the degrees of freedom corrected to more conservative values using the Huynh-Feldt $\varepsilon$ for any terms involving factors in which the sphericity assumption was violated. Significant main effects were further decomposed using pairwise comparisons with a Sidak correction.

In the SSRT task, four parameters were analyzed: SSRT, GoRT, stop accuracy, and go accuracy (no-delay trials). SSRTs were estimated based on the protocol described in Logan (1994), from the measures of baseline-corrected stoptrial accuracy and the distribution of go-trial RTs for each rat for each session. RTs on go trials were rank ordered and the $n$th RT selected from the ranked list of go trial RTs, where $n$ was obtained by multiplying the number of RTs in the distribution by the probability of responding on stop trials at that delay. The finishing time of the stop process was estimated by taking the $n$th fastest RT from a ranked set of GoRTs for each rat for each session. SSRT was calculated by subtracting stop-signal delay from the stop process finishing time. These procedures are described in detail in Eagle and Robbins (2003a). In the 5CSRTT, seven variables were analyzed: the percentage of correct responses made (number of correct responses/total correct and incorrect responses); percentage of responses omitted (number of omissions/total number of correct, incorrect, and omitted responses); percentage of premature responses (number of premature responses/total number of correct, incorrect, and omitted responses); latency to make a correct response; latency to make a premature response; latency to collect reward and perseverative nose pokes; and perseverative panel responses. In the delay-discounting paradigm, six variables were examined: percentage choice of the delayed reward, omissions, trial initiation latency, lever choice response latency, reward collection latency, and time spent nose poking during the delay to large reward.

\section{RESULTS}

\section{Experiment 1: Effect of Acute Atomoxetine on SSRT Performance}

Following analysis of inhibition functions, 11 rats were included in the drug study. Atomoxetine $(0.6-1.8 \mathrm{mg} / \mathrm{kg})$ induced a dose-dependent decrease in SSRT (Dose, $\mathrm{F}_{(4,36)}=5.1, p \leqslant 0.002$; Figure 1). Further pairwise comparisons revealed that both $1.0 \mathrm{mg} / \mathrm{kg}(p \leqslant 0.01)$ and $1.8 \mathrm{mg} / \mathrm{kg}$ $(p \leqslant 0.01)$ atomoxetine significantly reduced SSRT compared to vehicle controls. There was no significant difference in SSRT between vehicle and postdrug baseline $(p \leqslant 0.12)$, demonstrating that the above changes in SSRT were specific to treatment with atomoxetine, and not general improvements in SSRT over time. There was no overall difference between the fast-SSRT and slow-SSRT subgroups in their response to atomoxetine dose (GroupDose, $\mathrm{F}_{(4,36)}=1.5, p \leqslant 0.23$ ), however, a priori multiple comparisons revealed a significant decrease in SSRT for $1 \mathrm{mg} / \mathrm{kg}(p \leqslant 0.01)$ and $1.8 \mathrm{mg} / \mathrm{kg}(p \leqslant 0.01)$ atomoxetine in the slow-stopping animals compared to vehicle treatment in the same population, while there were no significant decreases in SSRT in the fast-stopping group. GoRT was not significantly altered by atomoxetine although there was a trend toward a dose-dependent lengthening (Dose, 


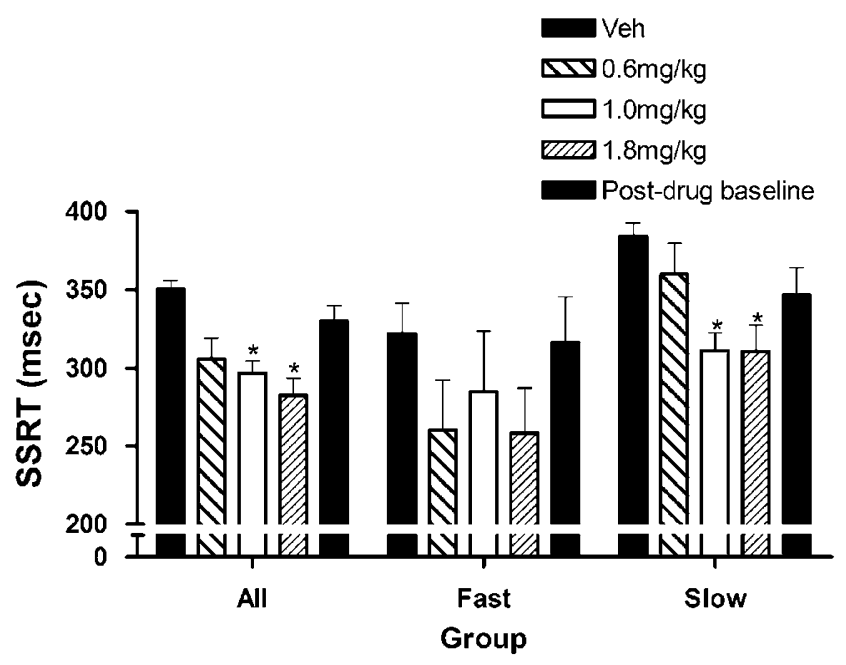

Figure I Effect of atomoxetine $(0.6-1.8 \mathrm{mg} / \mathrm{kg})$ on stop-signal reaction time (SSRT) in all rats and in fast and slow subgroupings. Atomoxetine $(1.0$ and $1.8 \mathrm{mg} / \mathrm{kg}$ ) decreases the SSRT in the whole group with a greater magnitude of decrease observed in slow-stopping animals compared with the fast-stopping subgroup. Vertical bars indicate the SEM (All $n=1 \mathrm{I}$, fast $n=6$, slow $n=5$ ). ${ }^{*} p<0.05$, Sidak-corrected pairwise comparison with vehicle control following significant main effect of atomoxetine dose.

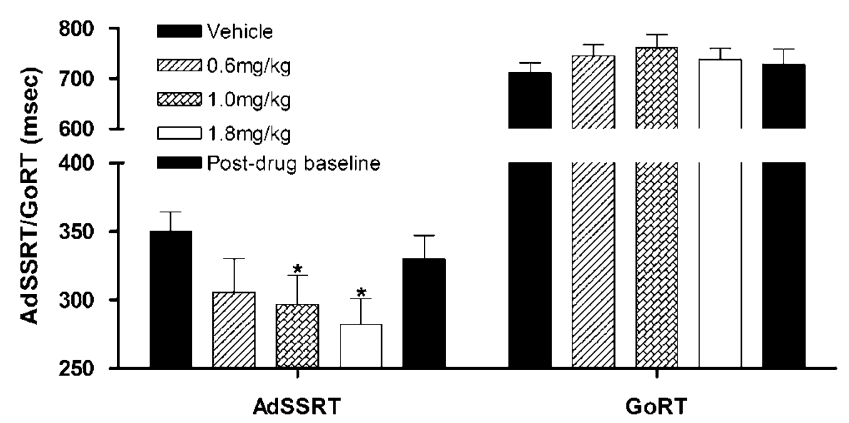

Figure 2 Effects of atomoxetine $(0.6-1.8 \mathrm{mg} / \mathrm{kg}$, i.p.) on stop-signal reaction time (SSRT) and go reaction time (GoRT) in the SSRT task for rats. Atomoxetine $(1.0$ and $1.8 \mathrm{mg} / \mathrm{kg}$ ) decreases the SSRT in the whole group while no significant effect on GoRT was observed. Vertical bars indicate the SEM $(n=1 \mathrm{I})$. * $p<0.05$, Sidak-corrected pairwise comparison with vehicle control following significant main effect of atomoxetine dose.

$\mathrm{F}_{(4,36)}=2.3, p \leqslant 0.08$; Figure 2$)$. No significant group $\times$ dose interaction for GoRT was observed (Table 1: Group $\times$ Dose $\left(\mathrm{F}_{(4,36)}=1.0, p \leqslant 0.44\right)$. Stop-trial performance accuracy on the task was not significantly altered by atomoxetine treatment (Table 1: Dose, $\mathrm{F}_{(4,36)}=2.1, p \leqslant 0.10$ ) and no significant group $\times$ dose interaction was observed (Table 1: Group $\times$ Dose, $\left.\mathrm{F}_{(4,36)}=1.1, p \leqslant 0.35\right)$, although the highest dose of atomoxetine appeared to reduce stop-trial accuracy in the fast-SSRT group while having no effect on the slowSSRT group. A significant main effect of atomoxetine dose on go accuracy was observed (Table 1: Dose, $\mathrm{F}_{(4,36)}=2.7$, $p \leqslant 0.05)$, however post hoc pairwise comparisons failed to reveal a significant difference between atomoxetine and vehicle control for any of the doses tested and between vehicle control and postdrug baseline. No significant group $\times$ dose interaction was observed (Group $\times$ Dose, $\left.\mathrm{F}_{(4,36)}=0.31, p \leqslant 0.87\right)$.
Experiment 2: Effect of Atomoxetine on the Five-Choice Serial Reaction Time Task

Following training and baseline stabilization, eight rats were included in the drug study. The effects of atomoxetine on 5CSRTT performance are summarized in Table 2. Atomoxetine $(0.6-3.0 \mathrm{mg} / \mathrm{kg}$, i.p.) induced a significant decrease in the percent premature responses $\left(\mathrm{F}_{(3,21)}=3.12, p \leqslant 0.05\right.$; Figure 3). Post hoc pairwise comparison revealed a significant effect at the $1 \mathrm{mg} / \mathrm{kg}$ dose $(p \leqslant 0.02)$. There was a strong trend toward a decrease in the percent correct responses made at the higher doses tested although there was no overall significant effect of dose $\left(F_{(3,21)}=2.59\right.$, $p \leqslant 0.08$; Figure 3$)$. No significant differences were observed for percent omissions $\left(\mathrm{F}_{(2,21)}=0.379, p \leqslant 0.63\right.$; Figure 3$)$ or perseverative responding (Table 2: perseverative nose pokes, $\mathrm{F}_{(2,21)}=1.63, p \leqslant 0.24$; perseverative panel pushes $\left.\mathrm{F}_{(2,21)}=0.441, p \leqslant 0.65\right)$. The latency to make a correct response (Table 2: $\mathrm{F}_{(3,14)}=1.47, p \leqslant 0.25$ ) or premature response (Table $2: \mathrm{F}_{(3,21)}=1.08, p \leqslant 0.38$ ) was not significantly different across doses. Collection latency was not significantly different although a trend toward an increase was observed (Table 2: $\mathrm{F}_{(2,14)}=2.8, p \leqslant 0.10$ ).

\section{Experiment 3: Effect of Atomoxetine on Delay Discounting}

From the original sample of 18 rats, 4 were excluded for not meeting the criterion of exhibiting delay sensitivity (see Materials and Methods). Two-factor within-subjects ANOVA of the choice data across the four drug administration sessions of the Latin-square design revealed that atomoxetine significantly increased the percentage choice for the larger, delayed reward (main effect, $\mathrm{F}_{(3,39)}=4.94, p \leqslant 0.005$; Figure 4). Further pairwise comparisons demonstrated that rats treated with $1 \mathrm{mg} / \mathrm{kg}$ atomoxetine displayed a significantly higher percentage choice for the delayed reward than vehicle-treated animals $(p \leqslant 0.02)$. There was a significant effect of delay on percentage choice of the delayed reward (main effect, $F_{(4,52)}=39.39, p<0.001$ ) where pairwise comparisons revealed increased choice of the delayed reward at 0 and $10 \mathrm{~s}$ as compared to choice at all longer delays (all $p$ 's $<0.01$ ). There was no significant dose $\times$ delay interaction for choice behavior $\left(\mathrm{F}_{(12,156)}=1.13, p \leqslant 0.34\right)$. In terms of general responding, performance was extremely reliable; only 3 of the 14 animals ever showed a rare (ie $\leqslant 2$ ) failure to initiate a trial via nose poke at a given delay, and no animals omitted a lever choice response once a trial had been initiated. No significant effects of atomoxetine dose were observed in trial initiation latency $\left(\mathrm{F}_{(3,39)}=0.45\right.$, $p \leqslant 0.72)$, lever choice latency $\left(\mathrm{F}_{(3,39)}=1.00, p \leqslant 0.40\right)$, reward collection latency $\left(\mathrm{F}_{(3,39)}=0.52, p \leqslant 0.67\right)$, or time spent nose poking during the delay to the large reward $\left(\mathrm{F}_{(3,18)}=0.05, p \leqslant 0.99\right)$.

\section{DISCUSSION}

This study has shown that atomoxetine reduces impulsive responding in three different animal models of impulsivity. Systemic atomoxetine $(1 \mathrm{mg} / \mathrm{kg})$ decreased (ie speeded) SSRT and reduced premature responding in the 5CSRTT, while it enhanced choice of the larger but delayed reward, 
Table I Results for the Effects of Atomoxetine on Performance Parameters in the Stop-Signal Reaction Time Task for Rats

\begin{tabular}{|c|c|c|c|c|c|}
\hline Parameter & Vehicle & $0.6 \mathrm{mg} / \mathrm{kg}$ & $1.0 \mathrm{mg} / \mathrm{kg}$ & $1.8 \mathrm{mg} / \mathrm{kg}$ & Postdrug baseline \\
\hline SSRT (ms) & $350.2 \pm 5.7$ & $305.5 \pm 13.5$ & $296.8 \pm 7.6 *$ & $282.3 \pm 11.1 *$ & $329.9 \pm 9.9$ \\
\hline Fast & $321.9 \pm 19.3$ & $260.3 \pm 31.9$ & $284.8 \pm 38.7$ & $258.7 \pm 28.5$ & $316.0 \pm 29.2$ \\
\hline Slow & $384.2 \pm 8.4$ & $359.8 \pm 20.0$ & $311.2 \pm 11.3^{*}$ & $310.6 \pm 16.5 *$ & $346.5 \pm 14.7$ \\
\hline GoRT (ms) & $712.1 \pm 4.4$ & $744.7 \pm 10.1$ & $761.7 \pm 15.6$ & $738.7 \pm 13.9$ & $728.3 \pm 12.4$ \\
\hline Correct stop (\%) & $83.2 \pm 2.0$ & $79.4 \pm 2.1$ & $78.7 \pm 2.5$ & $70.1 \pm 4.7$ & $79.3 \pm 3.1$ \\
\hline Fast & $77.0 \pm 6.0$ & $74.9 \pm 3.5$ & $75.4 \pm 4.8$ & $58.8 \pm 8.8$ & $74.7 \pm 5.9$ \\
\hline Slow & $90.6 \pm 3.0$ & $84.9 \pm 3.1$ & $82.5 \pm 3.6$ & $83.5 \pm 7.0$ & $84.8 \pm 4.7$ \\
\hline
\end{tabular}

Abbreviations: SSRT, stop-signal reaction time; GoRT, mean go reaction time.

Data are presented as the mean \pm SEM for the whole group $(n=11)$ and fast $(n=6)$ and slow $(n=5)$ SSRT subdivisions. Data were analyzed using repeated measures ANOVA with dose as the within-subject factor and group as a between-subjects factor.

${ }^{*} p<0.05$, Sidak-corrected pairwise comparison with vehicle control following significant main effect of atomoxetine dose.

Table 2 Results for the Effects of Atomoxetine on Performance Parameters in the Five-Choice Serial Reaction Time Task (5CSRTT) under Baseline Testing Conditions

\begin{tabular}{lcccc}
\hline Parameter & Vehicle & $\mathbf{0 . 6} \mathbf{~ m g / k g}$ & $\mathbf{1 . 0} \mathbf{~ m g / k g}$ & $\mathbf{3 . 0} \mathbf{~ m g / k g}$ \\
\hline Correct (\%) & $83.3 \pm 2.7$ & $85.3 \pm 2.5$ & $79.5 \pm 2.7$ & $80.2 \pm 2.1$ \\
Omissions (\%) & $5.3 \pm 2.7$ & $3.6 \pm 1.6$ & $7.3 \pm 5.1$ & $6.5 \pm 2.5$ \\
Premature (\%) & $13.8 \pm 2.1$ & $8.1 \pm 1.5$ & $6.3 \pm 2.1 *$ & $9.5 \pm 2.6$ \\
Persev. nose poke & $57.0 \pm 14.6$ & $58.3 \pm 14.5$ & $55.6 \pm 12.0$ & $42.3 \pm 7.8$ \\
Persev. panel push & $39.5 \pm 15.8$ & $38.1 \pm 18.0$ & $33.4 \pm 13.0$ & $42.4 \pm 15.1$ \\
Correct latency & $531.6 \pm 40.2$ & $564.3 \pm 36.3$ & $612.9 \pm 26.7$ & $599.7 \pm 37.6$ \\
Premature latency & $3988.3 \pm 3.0$ & $3614.8 \pm 377.1$ & $3718.3 \pm 336.3$ & $3842.3 \pm 404.2$ \\
Collection latency & $\mid 464.1 \pm 81.0$ & $1565.6 \pm 84.2$ & $1600.3 \pm 101.1$ & $1684 \pm 156.6$ \\
\hline
\end{tabular}

Abbreviation: Persev., perseverative.

Data are presented as the mean \pm SEM $(n=8)$. Data were analyzed using repeated measures ANOVA with dose as within-subject factor.

* $p<0.05$, Sidak-corrected pairwise comparison with vehicle control following significant main effect of atomoxetine dose.

indicating an ability to reduce both motor and choice impulsivity, considered to be fundamental components of disorders of behavioral inhibition, such as ADHD (Evenden, 1999). These results are therapeutically significant given that stimulants such as D-amphetamine and methylphenidate do not consistently reduce impulsivity in all of the above three tasks.

The effect of atomoxetine to improve SSRT is consistent with the results of Chamberlain et al (2006) for atomoxetine in healthy human volunteers performing the SSRT. In the rat SSRT task, atomoxetine is the first known example of a drug that is effective at reducing SSRT in rats independent of baseline performance: D-amphetamine, modafinil, and methylphenidate all show baseline-dependent effects on

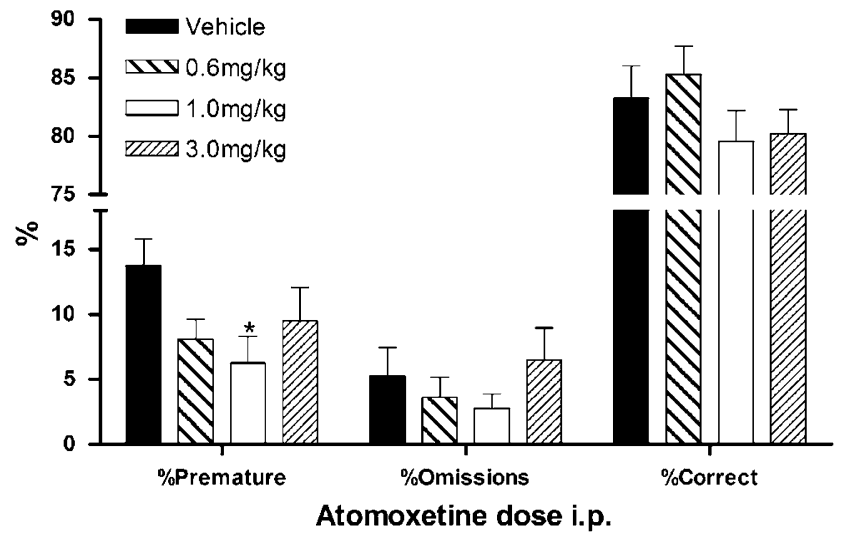

Figure 3 Effects of atomoxetine $(0.6-3 \mathrm{mg} / \mathrm{kg}$, i.p.) on the $5 \mathrm{CSRTT}$. Atomoxetine $(1.0 \mathrm{mg} / \mathrm{kg})$ decreased the percentage premature responses with no significant effects on response accuracy or omissions observed. Vertical bars indicate the SEM $(n=8)$. * $p<0.05$, Sidak-corrected pairwise comparison with vehicle control following significant main effect of atomoxetine dose.

SSRT (Feola et al, 2000; Eagle and Robbins, 2003a; Eagle et al, 2007). Therefore, unlike amphetamine, methylphenidate, and modafinil, atomoxetine can reduce impulsive responding in all animals on the SSRT task, an effect that is consistent with the results for premature responding in subpopulations of rats performing a 5CSRTT (Blondeau and Dellu-Hagedorn, 2006). GoRT was slightly slower following higher doses of atomoxetine, but this effect was not statistically significant, supporting the notion that the SSRTs and GoRTs are independently affected. Although there was a small increase in go accuracy no significant effects on stop accuracy were observed. This supports a selective effect of atomoxetine on the speed of stopping, rather than on activation of the stopping response per se, 


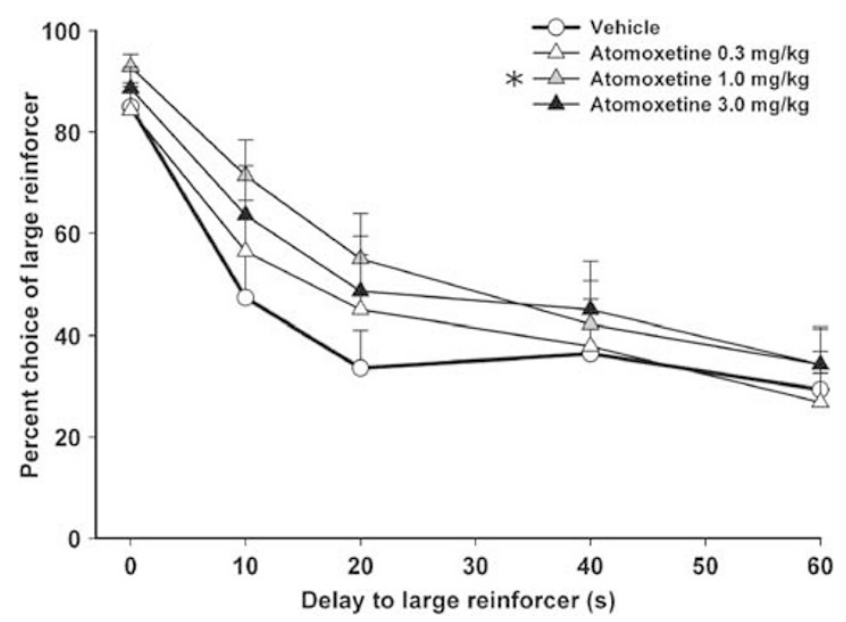

Figure 4 Effects of atomoxetine $(0.6-3.0 \mathrm{mg} / \mathrm{kg})$ on choice of a large, delayed reinforcer over a small, immediate reinforcer. Atomoxetine (I mg/ $\mathrm{kg}$ ) increases choice for the large, delayed reinforcer across the progressive delays. Vertical bars indicate the SEM $(n=14) . * p<0.05$, Sidak-corrected pairwise comparison with vehicle control following significant main effect of atomoxetine dose.

and confirms atomoxetine as a highly effective potential treatment for disorders in which slower SSRTs are the fundamental symptom.

Data from the 5CSRTT experiments showed that atomoxetine reduced premature responding at 0.6 and $1.0 \mathrm{mg} / \mathrm{kg}$ though no effect was observed for the highest dose $(3 \mathrm{mg} /$ $\mathrm{kg}$ ). The overall effect did not reach the same level of significance seen in the SSRT task or induce reductions at the low doses previously reported in a 5CSRTT (Blondeau and Dellu-Hagedorn, 2006). In their experiments, subcutaneous administration of atomoxetine reduced premature responding in a 5CSRTT in all groups but the effect was not significant for the group with the lowest baseline, which responded at a level comparable with the data from the present investigation. Consistent with the recent findings of Blondeau and Dellu-Hagedorn (2006), these data demonstrate that atomoxetine increases the animals' ability to withhold a response but does not affect other performance parameters such as accuracy.

In the final set of experiments, atomoxetine significantly increased the animals' choice of a larger but delayed reward over a smaller, immediate one. Unlike the SSRT or 5CSRTT, this paradigm does not depend on the animals inhibiting a prepotent motor response but rather challenges the animals' decision-making capacities to make adaptive choices (Evenden, 1999; Winstanley et al, 2006). All the doses tested tended to increase the animals' choice of the larger, delayed reward with a significant effect observed at $1 \mathrm{mg} / \mathrm{kg}$. The effect was observed as an upward shift in the delaydiscounting curve at the shorter delays of 10 and $20 \mathrm{~s}$, indicating that the animals were more likely to select the high-reward lever following atomoxetine treatment. In a previous study, the tricyclic antidepressant (TCA) drug, imipramine, had no effect on impulsive choice (Evenden and Ryan, 1996) while another study reported only small, inconsistent effects with the noradenaline-selective TCA, desipramine (DMI) (van Gaalen et al, 2006). Atomoxetine is more selective than the TCA and the lack of cholinergic and histaminergic effects seen with this drug may underlie the differences observed. Moreover, a larger impact on prefrontal NA is observed when DMI is administered chronically (Lapiz et al, 2006) and it is possible that the doses administered of acute DMI produced insufficiently effective increases in NA levels.

\section{Comparison with Other ADHD Medications}

The evaluation of ADHD medications using animal 'models' is not straightforward, since ADHD typically presents as a spectrum of disorders, and no animal 'models' replicate the full range of deficits found in ADHD (for discussion see Sagvolden et al, 2005; Russell et al, 2005). A number of previous studies in the SSRT task, 5CSRTT, and delaydiscounting paradigm have investigated the effects of ADHD medications on performance in normal populations. Amphetamine increases SSRT in poor performing humans and animals (de Wit et al, 2000; Eagle and Robbins, 2003a; Feola et al, 2000). Evaluation of both methylphenidate and modafinil in the SSRT task for rats revealed a similar profile to that observed for amphetamine with selective effects observed in slow-stopping animals (Eagle et al, 2007). Blockade of DA receptors using cis-flupenthixol did not block the effects of either methylphenidate or modafinil on stopping but did affect methylphenidate-induced changes in GoRT, suggesting the stop and go processes are under different neurochemical control (Eagle et al, 2007). This hypothesis is further supported by the present effects of atomoxetine on the SSRT task, for which SSRT-speeding effects again were independent of the GoRT.

Studies using the 5CSRTT have generally reported an increase in premature responding following administration of amphetamine or methylphenidate (Cole and Robbins, 1987, 1989; Harrison et al, 1997; Robbins, 2002). These effects appear contradictory when compared to data from the human literature where moderate doses of stimulants have been consistently shown to improve sustained attention and decrease impulsivity (Servan-Schreiber et al, 1990; Ward et al, 1997). It is, however, likely that these differences reflect the task that is used where amphetamine is proposed to counteract the effects of fatigue in vigilance situations (for discussion see Robbins, 2002). Although the majority of studies with stimulant drugs demonstrate increases in impulsivity, there have been reports suggesting that low doses of methylphenidate reduce impulsive responding in a poor performing subgroup of rats (Puumala et al, 1996). Low-dose oral methylphenidate has also been shown to reduce locomotor activity in rats suggesting that dose and route may be important factors in the response to stimulant medication (Kuczenski and Segal, 2002).

Premature responding in the 5CSRTT is altered by manipulations of the striatal DA system. In well-trained animals, stimulant drugs increase the vigor of responding with increased premature responses and decreased response latencies (Cole and Robbins, 1987, 1989). These effects have been shown to involve the nucleus accumbens (Cole and Robbins, 1989) and dorsal striatum, (Baunez and Robbins, 1999) respectively. In contrast to both amphetamine and methylphenidate, atomoxetine does not enhance DA release in the striatum (Bymaster et al, 2002). Consistent with these neurochemical studies, the ability of atomoxetine to reduce premature responding most likely reflects a lack of effect on 
striatal DA (Bymaster et al, 2002). Therefore, the effects of atomoxetine may reflect cortical $v s$ subcortical increases in catecholamines. Selective targeting of PFC catecholamine receptors has previously shown that activation of $\alpha_{2^{-}}$ adrenoceptors and $D_{1} D A$ receptors in the PFC contributes to the performance-enhancing effects of methylphenidate (Granon et al, 2000; Arnsten and Dudley, 2005).

Previous studies using amphetamine in healthy volunteers (de Wit et al, 2002) and rodents (Wade et al, 2000; Winstanley et al, 2003; van Gaalen et al, 2006) have shown increases in tolerance to delay in delay-discounting paradigms. Experiments in animals, using both amphetamine and methylphenidate, have shown decreased impulsivity, ie increased choice of a larger delayed reward over a smaller immediate reward (Evenden and Ryan, 1996; van Gaalen et $a l, 2006)$. A role for DA in mediating the effects of amphetamine and methylphenidate has been shown using selective drugs to block $\mathrm{D}_{1}$ or $\mathrm{D}_{2} \mathrm{DA}$ receptors (Wade et al, 2000; van Gaalen et al, 2006). The involvement of DA in these effects compared to NA has been further supported by studies using the selective DA reuptake inhibitor, GBR 12909, which mimics the effects seen with amphetamine and methylphenidate (van Gaalen et al, 2006). In contrast, blockade of NA reuptake using DMI had no effect (van Gaalen et al, 2006). These drugs were administered systemically therefore localization of the effects to a specific brain area could not be made.

ADHD has generally been considered a disease of DA hypofunction (for discussion see Solanto, 2002), however, a role for NA should not be excluded given that the medications currently used to treat ADHD target some component of the noradrenergic system (Biederman and Spencer, 1999; Arnsten, 2006a, b). Neurochemical studies with stimulant and nonstimulant medications have shown that atomoxetine, amphetamine, and methylphenidate all enhance DA and NA in the PFC (for review see Solanto, 1998; Arnsten and Li, 2005; Arnsten, 2006a,b) but atomoxetine does not increase DA in the nucleus accumbens (Bymaster et al, 2002). As such, a similarity in effects in the PFC is observed but the effects of stimulant and nonstimulant medications diverge in relation to subcortical structures (Bymaster et al, 2002; Swanson et al, 2006).

In summary, these findings demonstrate that atomoxetine reduces impulsive responding in behavioral tasks hypothesized to involve distinct neural processes (Evenden, 1999). Given the neurochemical profile of atomoxetine, these data suggest that a selective effect on cortical catecholamines may be an important mechanism of action on impulsivity. The lack of effect on subcortical DA may also account for the differences in behavioral effects observed in the present investigation when compared with results for stimulant drugs in the same tasks. The effects of atomoxetine also provide an important and significant finding in terms of its ability to consistently alter different types of impulsivity.

\section{ACKNOWLEDGEMENTS}

This research was supported by a programme grant from the Wellcome Trust to TWR, BJ Everitt, AC Roberts, and BJ Sahakian, and completed within University of Cambridge Behavioural and Clinical Neurosciences Institute (sup- ported by a joint award from the MRC and Wellcome Trust). ESJR holds an RCUK Academic Fellowship supported by the British Pharmacological Society's Integrative Pharmacology Fund. ACM holds a Fonds de la Recherche en Santé du Québec (FRSQ) postdoctoral fellowship. We thank David Theobald for technical assistance.

\section{DISCLOSURE/CONFLICT OF INTEREST}

The authors of this manuscript have no conflict of interests.

\section{REFERENCES}

Arnsten AF (2006a). Fundamentals of attention-deficit/hyperactivity disorder: circuits and pathways. J Clin Psychiatry Suppl 8: 7-12.

Arnsten AF (2006b). Stimulants: therapeutic actions in ADHD. Neuropsychopharmacology 31: 2376-2383.

Arnsten AF, Dudley AG (2005). Methylphenidate improves prefrontal cortical cognitive function through alpha2-adrenoceptor and dopamine D1 receptor actions: relevance to therapeutic effects in attention deficit hyperactivity disorder. Behav Brain Funct 1: 2.

Arnsten AF, Li BM (2005). Neurobiology of executive functions: catecholamine influences on prefrontal cortical functions. Biol Psychiatry 57: 1377-1384.

Aron AR, Dowson JH, Sahakian BJ, Robbins TW (2003b). Methylphenidate improves response inhibition in adults with attention-deficit/hyperactivity disorder. Biol Psychiatry 54: 1465-1468.

Aron AR, Fletcher PC, Bullmore ET, Sahakian BJ, Robbins TW (2003a). Stop-signal inhibition disrupted by damage to right inferior frontal gyrus in humans. Nat Neurosci 6: 115-116.

Badcock JC, Michie PT, Johnson L, Combrinck J (2002). Acts of control in schizophrenia: dissociating the components of inhibition. Psychol Med 32: 287-297.

Baunez C, Robbins TW (1999). Effects of dopamine depletion of the dorsal striatum and further interaction with subthalamic nucleus lesions in an attentional task in the rat. Neurosci 92: 1343-1356.

Berridge CW, Devilbiss DM, Andrzejewski ME, Arnsten AF, Kelley AE, Schmeichel B et al (2006). Methylphenidate preferentially increases catecholamine neurotransmission within the prefrontal cortex at low doses that enhance cognitive function. Biol Psychiatry 60: 1111-1120.

Biederman J, Spencer T (1999). Attention-deficit/hyperactivity disorder (ADHD) as a noradrenergic disorder. Biol Psychiatry 46: $1234-1242$.

Bizot JC (1998). Effects of various drugs including organophosphorus compounds (OPC) and therapeutic compounds against OPC on DRL responding. Pharmacol Biochem Behav 59: 1069-1080.

Blondeau C, Dellu-Hagedorn F (2006). Dimensional analysis of ADHD subtypes in rats. Biol Psychiatry 61: 1340-1350.

Boonstra AM, Kooij JJ, Oosterlaan J, Sergeant JA, Buitelaar JK (2005). Does methylphenidate improve inhibition and other cognitive abilities in adults with childhood-onset ADHD? J Clin Exp Neuropsychol 27: 278-298.

Bymaster FP, Katner JS, Nelson DL, Hemrick-Luecke SK, Threlkeld PG, Heiligenstein JH et al (2002). Atomoxetine increases extracellular levels of norepinephrine and dopamine in prefrontal cortex of rat: a potential mechanism for efficacy in attention deficit/hyperactivity disorder. Neuropsychopharmacology 27: 699-711.

Cardinal RN, Aitken MRF (2001). Whisker, version 2.2. http:// www.whiskercontrol.com. 
Cardinal RN, Pennicott DR, Sugathapala CL, Robbins TW, Everitt BJ (2001). Impulsive choice induced in rats by lesions of the nucleus accumbens core. Science 292: 2499-2501.

Cardinal RN, Robbins TW, Everitt BJ (2000). The effects of d-amphetamine, chlordiazepoxide, alpha-flupenthixol and behavioral manipulations on choice of signalled and unsignalled delayed reinforcement in rats. Psychopharmacology 152: 362-375.

Carli M, Robbins TW, Evenden JL, Everitt BJ (1983). Effects of lesions to ascending noradrenergic neurones on performance of a 5-choice serial reaction task in rats; implications for theories of dorsal noradrenergic bundle function based on selective attention and arousal. Behav Brain Res 9: 361-380.

Chamberlain SR, Muller U, Blackwell AD, Clark L, Robbins TW, Sahakian BJ (2006). Neurochemical modulation of response inhibition and probabilistic learning in humans. Science 311: 861-863.

Cole BJ, Robbins TW (1987). Amphetamine impairs the discriminative performance of rats with dorsal noradrenergic bundle lesions on a 5-choice serial reaction time task: new evidence for central dopaminergic-noradrenergic interactions. Psychopharmacology 91: 458-466.

Cole BJ, Robbins TW (1989). Effects of 6-hydroxydopamine lesions of the nucleus accumbens septi on performance of a 5 -choice serial reaction time task in rats: implications for theories of selective attention and arousal. Behav Brain Res 33: 165-179.

Dalley JW, Cardinal RN, Robbins TW (2004). Prefrontal executive and cognitive functions in rodents: neural and neurochemical substrates. Neurosci Biobehav Rev 28: 771-784.

de Wit H, Crean J, Richards JB (2000). Effects of d-amphetamine and ethanol on a measure of behavioral inhibition in humans. Behav Neurosci 114: 830-837.

de Wit H, Enggasser JL, Richards JB (2002). Acute administration of d-amphetamine decreases impulsivity in healthy volunteers. Neuropsychopharmacology 27: 813-825.

Eagle DM, Baunez C, Hutcheson DM, Lehmann O, Shah AP, Robbins TW (2007a). Stop-signal reaction time task performance: role of prefrontal cortex and subthalamic nucleus. Cerebral Cortex (doi:10.1093/cercor/bhm044).

Eagle DM, Robbins TW (2003a). Inhibitory control in rats performing a stop-signal reaction-time task: effects of lesions of the medial striatum and d-amphetamine. Behav Neurosci 117: 1302-1317.

Eagle DM, Robbins TW (2003b). Lesions of the medial prefrontal cortex or nucleus accumbens core do not impair inhibitory control in rats performing a stop-signal reaction time task. Behav Brain Res 146: 131-144.

Eagle DM, Tufft MRA, Goodchild HL, Robbins TW (2007b). Differential effects of modafinil and methylphenidate on stopsignal reaction time task performance in the rat, and interactions with the dopamine receptor antagonist cis-flupenthixol. Psychopharmacology 192: 193-206.

Evenden JL (1999). The pharmacology of impulsive behavior in rats VII: the effects of serotonergic agonists and antagonists on responding under a discrimination task using unreliable visual stimuli. Psychopharmacology 146: 422-431.

Evenden JL, Ryan CN (1996). The pharmacology of impulsive behavior in rats: the effects of drugs on response choice with varying delays of reinforcement. Psychopharmacology 128: 161-170.

Feola TW, de Wit H, Richards JB (2000). Effects of d-amphetamine and alcohol on a measure of behavioral inhibition in rats. Behav Neurosci 114: 838-848.

Fillmore MT, Rush CR (2002). Impaired inhibitory control of behavior in chronic cocaine users. Drug Alcohol Depend 66: 265-273.
Gauggel S, Rieger M, Feghoff TA (2004). Inhibition of ongoing responses in patients with Parkinson's disease. I Neurol Neurosurg Psychiatry 75: 539-544.

Granon S, Passetti F, Thomas KL, Dalley JW, Everitt BJ, Robbins TW (2000). Enhanced and impaired attentional performance after infusion of D1 dopaminergic receptor agents into rat prefrontal cortex. J Neurosci 20: 1208-1215.

Harrison AA, Everitt BJ, Robbins TW (1997). Central 5-HT depletion enhances impulsive responding without affecting the accuracy of attentional performance: interactions with dopaminergic mechanisms. Psychopharmacology 133: 329-342.

Kratochvil CJ, Vaughan BS, Harrington MJ, Burke WJ (2003). Atomoxetine: a selective noradrenaline reuptake inhibitor for the treatment of attention-deficit/hyperactivity disorder. Expert Opin Pharmacother 4: 1165-1174.

Kratochvil CJ, Wilens TE, Greenhill LL, Gao H, Baker KD, Feldman PD et al (2006). Effects of long-term atomoxetine treatment for young children with attention-deficit/hyperactivity disorder. J Am Acad Child Adolesc Psychiatry 45: 919-927.

Krikorian R, Zimmerman ME, Fleck DE (2004). Inhibitory control in obsessive-compulsive disorder. Brain Cogn 54: 257-259.

Kuczenski R, Segal DS (2002). Exposure of adolescent rats to oral methylphenidate: preferential effects on extracellular norepinephrine and absence of sensitization and cross-sensitization to methamphetamine. J Neurosci 22: 7264-7271.

Lapiz MD, Bondi CO, Morilak DA (2006). Chronic treatment with desipramine improves cognitive performance of rats in an attentional set-shifting test. Neuropsychopharmacology 32: $1000-1010$.

Logan GD (1994). On the ability to inhibit thought and action. A users' guide to the stop signal paradigm. In: Dagenbach D, Carr $\mathrm{TH}$ (eds). Inhibitory Processes in Attention, Memory and Language. Academic Press: San Diego. pp 189-236.

Logan GD, Cowan WB (1984). On the ability to inhibit thought and action - a theory of an act of control. Psychol Rev 91: 295-327.

Mar AC, Robbins TW (2007). Delay discounting and impulsive choice in the rat. Curr Protoc Neurosci 8.22: 1-18.

Monterosso JR, Aron AR, Cordova X, Xu J, London ED (2005). Deficits in response inhibition associated with chronic methamphetamine abuse. Drug Alcohol Depend 79: 273-277.

Puumala T, Ruotsalainen S, Jakala P, Koivisto E, Riekkinen Jr P, Sirvio J (1996). Behavioral and pharmacological studies on the validation of a new animal model for attention deficit hyperactivity disorder. Neurobiol Learn Mem 66: 198-211.

Richards JB, Sabol KE, de Wit H (1999). Effects of methamphetamine on the adjusting amount procedure, a model of impulsive behavior in rats. Psychopharmacology 146: 432-439.

Robbins TW (2002). The 5-choice serial reaction time task: behavioral pharmacology and functional neurochemistry. Psychopharmacology 163: 362-380.

Rubia K (2002). The dynamic approach to neurodevelopmental psychiatric disorders: use of fMRI combined with neuropsychology to elucidate the dynamics of psychiatric disorders, exemplified in ADHD and schizophrenia. Behav Brain Res 130: 47-56.

Rubia K, Russell T, Bullmore ET, Soni W, Brammer MJ, Simmons A et al (2001). An fMRI study of reduced left prefrontal activation in schizophrenia during normal inhibitory function. Schizophr Res 52: 47-55.

Rubia K, Smith AB, Brammer MJ, Taylor E (2003). Right inferior prefrontal cortex mediates response inhibition while mesial prefrontal cortex is responsible for error detection. Neuroimage 20: 351-358.

Russell VA, Sagvolden T, Johansen EB (2005). Animal models of attention-deficit hyperactivity disorder. Behav Brain Func 1: 9.

Sagvolden T, Russell VA, Aase H, Johansen EB, Farshbaf M (2005). Rodent models of attention-deficit/hyperactivity disorder. Biol Psychiatry 57: 1239-1247. 
Servan-Schreiber D, Printz H, Cohen JD (1990). A network model of catecholamine effects: gain, signal-to-noise ratio, and behavior. Science 249: 892-895.

Solanto MV (1998). Neuropsychopharmacological mechanisms of stimulant drug action in attention-deficit hyperactivity disorder: a review and integration. Behav Brain Res 94: 127-152.

Solanto MV (2002). Dopamine dysfunction in AD/HD: integrating clinical and basic neuroscience research. Behav Brain Res 130: $65-71$.

Swanson CJ, Perry KW, Koch-Krueger S, Katner J, Svensson KA, Bymaster FP (2006). Effect of the attention deficit/hyperactivity disorder drug atomoxetine on extracellular concentrations of norepinephrine and dopamine in several brain regions of the rat. Neuropharmacology 50: 755-760.

Tannock R, Schachar RJ, Carr RP, Chajczyk D, Logan GD (1989). Effects of methylphenidate on inhibitory control in hyperactive children. J Abnorm Child Psychol 17: 473-491.

Turner DC, Clark L, Dowson J, Robbins TW, Sahakian BJ (2004). Modafinil improves cognition and response inhibition in adult attention-deficit/hyperactivity disorder. Biol Psychiatry 55: 1031-1040.

Turner DC, Robbins TW, Clark L, Aron AR, Dowson J, Sahakian BJ (2003). Cognitive enhancing effects of modafinil in healthy volunteers. Psychopharmacology 165: 260-269. van Gaalen MM, Brueggeman RJ, Bronius PF, Schoffelmeer AN, Vanderschuren LJ (2006). Behavioral disinhibition requires dopamine receptor activation. Psychopharmacology 187: 73-85.

Wade TR, de Wit H, Richards JB (2000). Effects of dopaminergic drugs on delayed reward as a measure of impulsive behavior in rats. Psychopharmacology 150: 90-101.

Ward AS, Kelly TH, Foltin RW, Fischman MW (1997). Effects of d-amphetamine on task performance and social behavior of humans in a residential laboratory. Exp Clin Psychopharmacol 5: 130-136.

Wilens TE (2006). Mechanism of action of agents used in attention-deficit/hyperactivity disorder. J Clin Psychiatry 67(Suppl 8): 32-38.

Winstanley CA, Dalley JW, Theobald DE, Robbins TW (2003). Global 5-HT depletion attenuates the ability of amphetamine to decrease impulsive choice on a delay-discounting task in rats. Psychopharmacology 170: 320-331.

Winstanley CA, Eagle DM, Robbins TW (2006). Behavioral models of impulsivity in relation to ADHD: translation between clinical and preclinical studies. Clin Psychol Rev 26: 379-395.

Winstanley CA, Theobald DE, Dalley JW, Robbins TW (2005). Interactions between serotonin and dopamine in the control of impulsive choice in rats: therapeutic implications for impulse control disorders. Neuropsychopharmacology 30: 669-682. 